
\title{
AIP
}

\section{Anomalous Hall effect and magnetoresistance behavior in Co/Pd1-xAgx multilayers}

Z. B. Guo, W. B. Mi, A. Manchon, J. Q. Li, B. Zhang, P. G. Barba, and X. X. Zhang

Citation: Applied Physics Letters 102, 062413 (2013); doi: 10.1063/1.4792054

View online: http://dx.doi.org/10.1063/1.4792054

View Table of Contents: http://scitation.aip.org/content/aip/journal/apl/102/6? ver=pdfcov

Published by the AIP Publishing

\section{Articles you may be interested in}

Anomalous Hall effect in $\mathrm{Co} / \mathrm{Ni}$ multilayers with perpendicular magnetic anisotropy

Appl. Phys. Lett. 104, 082404 (2014); 10.1063/1.4866774

Effect of interfacial structures on anomalous Hall behavior in perpendicular Co/Pt multilayers

Appl. Phys. Lett. 102, 102404 (2013); 10.1063/1.4795331

Current driven oscillation and switching in Co/Pd perpendicular giant magnetoresistance multilayer

J. Appl. Phys. 109, 07 C905 (2011); 10.1063/1.3540415

Magnetoresistive effects in Co/Pd multilayers on self-assembled nanoparticles (invited)

J. Appl. Phys. 107, 09 C506 (2010); 10.1063/1.3350909

Reversal of the extraordinary Hall effect polarity in thin Co/Pd multilayers

Appl. Phys. Lett. 96, 022512 (2010); 10.1063/1.3291707

You don't

still use this

cell phone

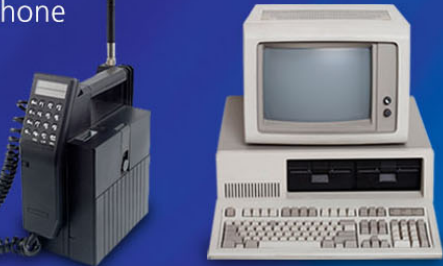

Why are you still using an AFM designed in the 80 's?
It is time to upgrade your AFM

Minimum $\$ 20,000$ trade-in discount for purchases before August 31st

Asylum Research is today's technology leader in AFM

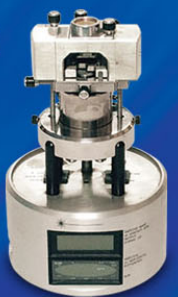




\title{
Anomalous Hall effect and magnetoresistance behavior in $\mathrm{Co} / \mathrm{Pd}_{1-\mathrm{x}} \mathbf{A g}_{\mathrm{x}}$ multilayers
}

\author{
Z. B. Guo, ${ }^{1}$ W. B. Mi, ${ }^{2}$ A. Manchon, ${ }^{3}$ J. Q. Li, ${ }^{1}$ B. Zhang, ${ }^{1}$ P. G. Barba,${ }^{3}$ and X. X. Zhang ${ }^{1, a)}$ \\ ${ }^{1}$ Core Labs, King Abdullah University of Science and Technology (KAUST), Thuwal 23955-6900, Saudi Arabia \\ ${ }^{2}$ Tianjin Key Laboratory of Low Dimensional Materials Physics and Preparing Technology, Institute of \\ Advanced Materials Physics, Faculty of Science, Tianjin University, Tianjin 300072, China \\ ${ }^{3}$ Materials Science and Engineering, King Abdullah University of Science and Technology (KAUST), \\ Thuwal 23955-6900, Saudi Arabia
}

(Received 19 November 2012; accepted 30 January 2013; published online 13 February 2013)

In this paper, we report anomalous Hall effect (AHE) correlated with the magnetoresistance behavior in $\left[\mathrm{Co} / \mathrm{Pd}_{1-x} \mathrm{Ag}_{x}\right]_{n}$ multilayers. For the multilayers with $n=6$, the increase in $\mathrm{Ag}$ content from $x=0$ to 0.52 induces the change in AHE sign from negative surface scattering-dominated AHE to positive interface scattering-dominated AHE, which is accompanied with the transition from anisotropy magnetoresistance (AMR) dominated transport to giant magnetoresistance (GMR) dominated transport. For $n=80$, scaling analysis with $R_{s} \propto \rho_{x x}^{\gamma}$ yields $\gamma \sim 3.44$ for $x=0.52$ which presents GMR-type transport, in contrast to $\gamma \sim 5.7$ for $x=0$ which presents AMR-type transport. (C) 2013 American Institute of Physics. [http://dx.doi.org/10.1063/1.4792054]

Anomalous Hall effect (AHE) in magnetic materials was discovered more than a century ago. ${ }^{1}$ Recently, the intensive interest in AHE has been promoted by its spindependent transport properties and technical applications. ${ }^{2-4}$ The Hall resistivity $\left(\rho_{H}\right)$ was found to follow the form of the phenomenological equation

$$
\rho_{H}=R_{o} B+4 \pi R_{s} M,
$$

where the first term is the ordinary Hall resistivity due to the Lorentz force and the second term is anomalous Hall resistivity $\left(\rho_{A H}\right)$, which is proportional to the out-of-plane magnetization of the film. ${ }^{4}$ To identify the origins of AHE, the scaling correlation between anomalous Hall coefficient $R_{s}$ in Eq. (1) and zero-field longitudinal resistivity $\rho_{x x}$ given by

$$
R_{s} \propto \rho_{x x}^{\gamma}
$$

is usually employed. ${ }^{2}$ Skew scattering mechanism leads to the exponent $\gamma=1,5$ while both scattering dependent-side jump and scattering independent-intrinsic mechanism yield $\gamma=2{ }^{6-8}$ For homogeneous ferromagnets, experimental $\gamma$ values are in the range of $1-2$, demonstrating the general coexistence of these mechanisms. ${ }^{9,10}$ However, for heterogeneous ferromagnetic systems, such as magnetic multilayers and granular films, interface scattering-dominated AHE has been proven to result in a large $\gamma$, for example, $\gamma=2.6$ in $\mathrm{Fe} / \mathrm{Cr}$ multilayers, ${ }^{11} \gamma=2.6$ and 5.7 in $\mathrm{Co} / \mathrm{Pd}$ multilayers, ${ }^{12,13}$ and $\gamma=3.7$ in granular Co-Ag films, ${ }^{14}$ while the mechanism of interface scattering on the value of $\gamma$ is still missing in theory. On the other hand, in single layer films, such as $\mathrm{Ni}$, surface scattering has been found to play a dominant role on AHE for film thickness less than $50 \mathrm{~nm} .{ }^{15,16}$ In ferromagnetic/nonmagnetic (FM/NM) multilayers, the sign of surface scatteringinduced AHE has been found to be opposite to that of

\footnotetext{
${ }^{\text {a) }}$ Author to whom correspondence should be addressed. Electronic mail: xixiang.zhang@kaust.edu.sa.
}

interface scattering in $\mathrm{Co} / \mathrm{Pd}$ multilayers, and the amplitude of the former could even overwhelm the latter. ${ }^{13,17}$

In this paper, we studied AHE and magnetoresistance (MR) behavior in $\left[\mathrm{Co}(3 \AA) / \mathrm{Pd}_{1-x} \mathrm{Ag}_{x}(5 \AA)\right]_{n}$ multilayers with perpendicular magnetic anisotropy. With the increase of the substitution of $\mathrm{Ag}$, the sign change in AHE accompanied with the increase of giant magnetoresistance (GMR) has been observed in the multilayers with $n=6$, which should be attributed to the transition from surface scattering-dominated AHE to interface scattering-dominated AHE.

Two series of $\mathrm{Co} / \mathrm{Pd}_{1-x} \mathrm{Ag}_{x}$ multilayer with a structure of $\mathrm{Pd}_{1-x} \operatorname{Ag}_{x}(50 \AA) /\left[\mathrm{Co}(3 \AA) / \mathrm{Pd}_{1-x} \operatorname{Ag}_{x}(5 \AA)\right]_{n}$ were deposited by using magnetron sputtering on oxidized $\mathrm{Si}$ (100) substrates, with the $\mathrm{Co} / \mathrm{PdAg}$ bilayers' repetition $n$ of 6 and 80 . A 10-nm-thick $\mathrm{SiO}_{2}$ was deposited to cover the films to prevent from oxidation. The $\mathrm{Pd}_{1-x} \mathrm{Ag}_{x}$ layers were deposited by co-sputtering, where the target power for Pd was fixed, by the means of varying the target power of $\mathrm{Ag}$ to adjust the $\mathrm{Ag}$ content. The $\mathrm{Ag}$ contents of $x=0,0.04,0.19,0.32$, and 0.52 were obtained, which were measured by energy-dispersive spectrometer on relatively thick $\mathrm{Co}_{1-x} \mathrm{Ag}_{x}$ films, larger than $200 \mathrm{~nm}$. Hall loops were measured under magnetic fields up to $5 \mathrm{~T}$ applied perpendicular to the film plane. Detailed Hall bar fabrication and measurement can be found in Ref. 13.

Bogomolov et al. have studied the magnetic properties and structures of $\mathrm{Co}(4 \AA) / \mathrm{Pd}_{1-\mathrm{x}} \mathrm{Ag}_{\mathrm{x}}(10 \AA)$ multilayers. ${ }^{18} \mathrm{~A}$ significant increase in coercivity $\left(\mathrm{H}_{\mathrm{c}}\right)$ from $1.4 \mathrm{kOe}$ in $x=0$ to $2.7 \mathrm{kOe}$ in $x=0.04$ has been observed. The origin of the increase in $\mathrm{H}_{\mathrm{c}}$ by substituting Pd with $\mathrm{Ag}$ has been attributed to the increase of tensile stress because of the larger lattice constant of face-centered cubic (fcc) $\mathrm{Ag}(\sim 4.086 \AA)$ than that of fcc Pd $(\sim 3.883 \AA)$. Further increase of the substitution results in a decrease in $\mathrm{H}_{c}$, due to the decrease in the multilayer lattice matching, forming interface misfit dislocations. ${ }^{18}$

In this paper, we focused on magnetotransport properties in terms of $\mathrm{AHE}$ and $\mathrm{MR}$ in $\mathrm{Co} / \mathrm{Pd}_{1-x} \mathrm{Ag}_{x}$ multilayers. Shown in Fig. 1 are the representative Hall loops of $[\mathrm{Co} /$ $\left.\mathrm{Pd}_{1-\mathrm{x}} \mathrm{Ag}_{\mathrm{x}}\right]_{6}$ multilayer with $x=0,0.04,0.19$, and 0.32 
measured at $5 \mathrm{~K}$. All the samples present perpendicular magnetic anisotropy as square hysteresis loops could be obtained after removal of the linear background of ordinary Hall resistivity. $\mathrm{H}_{\mathrm{c}}$ increases from $5.4 \mathrm{kOe}$ for $x=0$ to $10.7 \mathrm{kOe}$ for $x=0.04$. Considerably larger $\mathrm{H}_{\mathrm{c}}$ in our samples than that of the samples of Bogomolov et al. should be attributed to the thinner Co layers in our samples, which demonstrates the nature of interface-induced perpendicular magnetic anisotropy in this structure. ${ }^{19,20} \mathrm{H}_{\mathrm{c}}$ decreases as $x$ increases to more than 0.19 . The dependence of $H_{c}$ on $x$ is in good agreement with that observed by Bogomolov et al. ${ }^{18}$

The AHE resistivity $\rho_{A H}$ can be extracted from the Hall loops by extrapolating the high-field data from positive field to zero field, as shown in Fig. 1. The sign of AHE is negative for $x \leq 0.19$, while it is positive for $x \geq 0.32$, indicating the change in AHE sign with the substitution of Pd with $\mathrm{Ag}$. Later analysis indicates the sign change from negative to positive is due to the transition from negative surface scattering-dominated AHE to positive interface scatteringdominated AHE.

The temperature dependence of $\rho_{A H}$ and $\rho_{x x}$ for $[\mathrm{Co} /$ $\left.\mathrm{Pd}_{1-x} \mathrm{Ag}_{x}\right]_{6}$ multilayers is shown in Figs. 2(a) and 2(b), respectively. For $x=0$, the negative $\rho_{A H}$ and the decrease in the amplitude of $\left|\rho_{A H}\right|$ with increasing temperature have been attributed to surface scattering-dominated AHE. ${ }^{13}$ These characteristics are also presented in the multilayers with $x=0.04$ and 0.19 . However, for $x=0.32$ and $0.52, \rho_{A H}$ is positive, as can be seen clearly from the enlarged view in the inset in Fig. 2(a). $\rho_{A H}$ increases with raising temperature up to $250 \mathrm{~K}$ for $x=0.32$ and in the whole temperature range for $x=0.52$. The increase of $\rho_{A H}$ with increasing temperature is a typical behavior of bulk/interface scatteringdominated AHE. The decrease in $\rho_{A H}$ for temperature above $250 \mathrm{~K}$ for $x=0.32$ implies the positive AHE from surface scattering, which is suppressed with increasing temperature due to the decrease in mean free path.

The temperature dependence of longitudinal resistivity $\rho_{x x}$ for $\left[\mathrm{Co} / \mathrm{Pd}_{1-x} \mathrm{Ag}_{x}\right]_{6}$ multilayers is shown in Fig. 2(b), which indicates that all the films are metallic. The overall tendency is that $\rho_{x x}$ decreases with increasing $x$, except an abnormal increase in the film with $x=0.19$. For this film, it

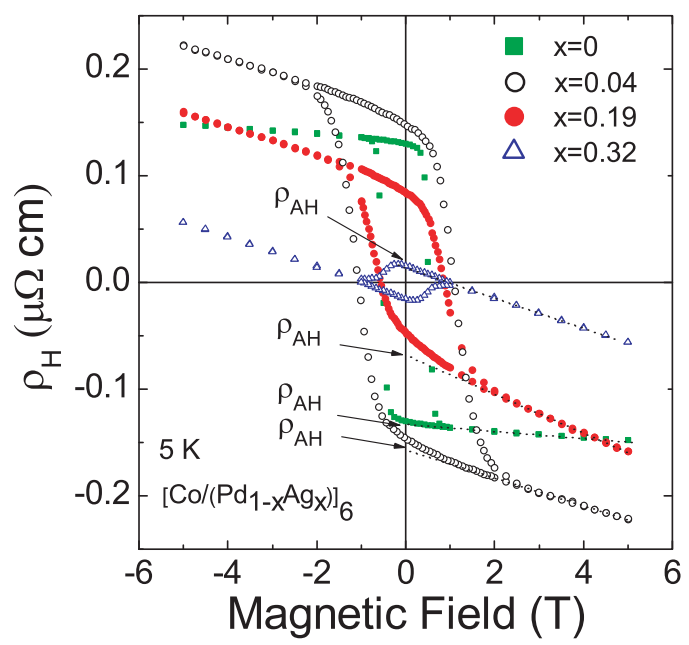

FIG. 1. The representative Hall loops of $\left[\mathrm{Co}(3 \AA) / \operatorname{Pd}_{1-x} \operatorname{Ag}_{x}(5 \AA)\right]_{6}$ multilayers with $\mathrm{x}=0,0.04,0.19$, and 0.32 measured at $5 \mathrm{~K}$.

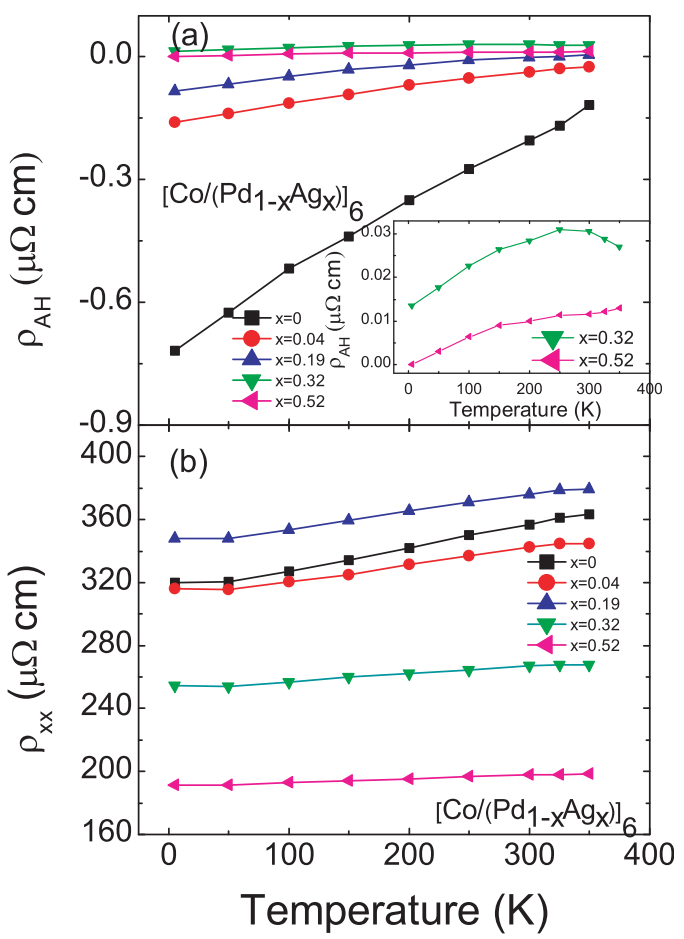

FIG. 2. The temperature dependence of (a) $\rho_{A H}$ and (b) $\rho_{x x}$ for the $[\operatorname{Co}(3 \AA) /$ $\left.\mathrm{Pd}_{1-x} \operatorname{Ag}_{x}(5 \AA)\right]_{6}$ multilayers with $x=0,0.04,0.19,0.32$, and 0.52 , respectively. Inset in (a): enlarged view for $x=0.32$ and 0.52 .

also shows a smaller $\mathrm{H}_{\mathrm{c}}$ than that of $x=0.04$, in contrast to the increase in $\mathrm{H}_{\mathrm{c}}$ with increasing $x$ from 0 to 0.04 (Fig. 1). Therefore, the increase in $\rho_{x x}$ in $x=0.19$ could be related to the interface misfit dislocations.

Shown in Fig. 3 are the representative MR curves of $\left[\mathrm{Co} / \mathrm{Pd}_{1-x} \mathrm{Ag}_{x}\right]_{6}$ multilayers measured at $5 \mathrm{~K}$ with magnetic field applied in the film plane, where $\rho_{\|}$corresponds to the magnetic field parallel to the current direction and $\rho_{\perp}$ for the field perpendicular to the current direction. The multilayer with $x=0$ exhibits an AMR behavior [Fig. 3(a)], and the multilayer with $x=0.04$ presents both AMR and GMR behaviors, where the AMR behavior is embodied in the obvious difference between $\rho_{\|}$and $\rho_{\perp}$ at $5 \mathrm{~T}$ [Fig. 3(b)]. For $x=0.52$, the multilayer presents a GMR-dominated behavior [Fig. 3(c)].

GMR ratio and $\rho_{A H}$ as a function of $x$ at $5 \mathrm{~K}$ for $[\mathrm{Co} /$ $\left.\mathrm{Pd}_{1-x} \mathrm{Ag}_{x}\right]_{6}$ multilayers with $x=0.04,0.19,0.32$, and 0.52 are shown in Figs. 4(a) and 4(b), respectively, where the GMR ratio is defined by GMR ratio $=\left[\rho_{\max }-\rho(5 \mathrm{~T})\right] / \rho(5 \mathrm{~T}), \rho_{\max }$ is the maximum $\rho_{x x}$ in $\rho_{\|}$, and $\rho(5 \mathrm{~T})$ is the value of $\rho_{\|}$at $5 \mathrm{~T}$. In Fig. 4(a), one can find a sharp increase in GMR ratio as $x$ increases from 0.19 to 0.32 , where the sign of AHE crossovers from negative to positive, i.e., from surface scatteringdominated AHE to interface scattering-dominated AHE. It is well known that GMR effect is related to spin-dependent interface scattering in FM/NM multilayers. In GMR theory, the mechanism of GMR and amplitude of GMR ratio are correlated with the mean free path and spin-dependent coefficients at the interfaces for specular reflection, transmission, and diffuse scattering. ${ }^{21,22}$ The gradual substitution of $\mathrm{Pd}$ by $\mathrm{Ag}$ in our experiment, which modifies these parameters, enables us to observe the transition in magnetoresistance from AMR-dominated to GMR-dominated, correlated with the 


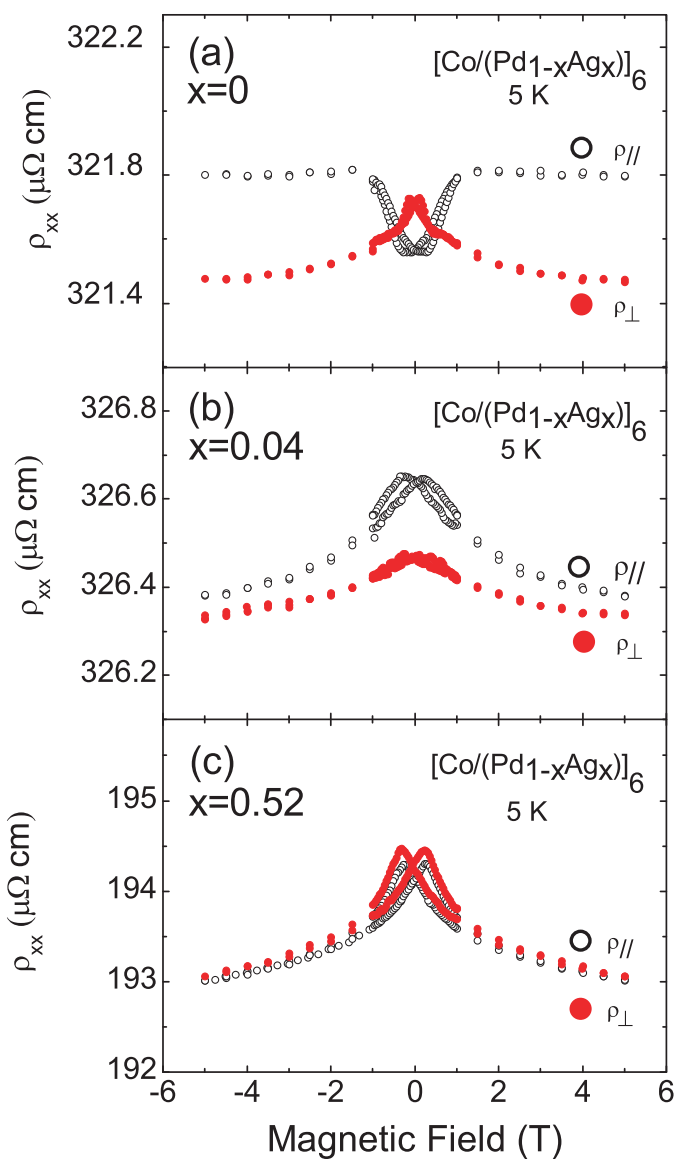

FIG. 3. The representative MR curves of $\left[\mathrm{Co}(3 \AA) / \operatorname{Pd}_{1-x} \operatorname{Ag}_{x}(5 \AA)\right]_{6}$ multilayers for (a) $x=0$, (b) $x=0.04$, and (c) $x=0.52$ measured at $5 \mathrm{~K}$ with magnetic field applied in the film plane.

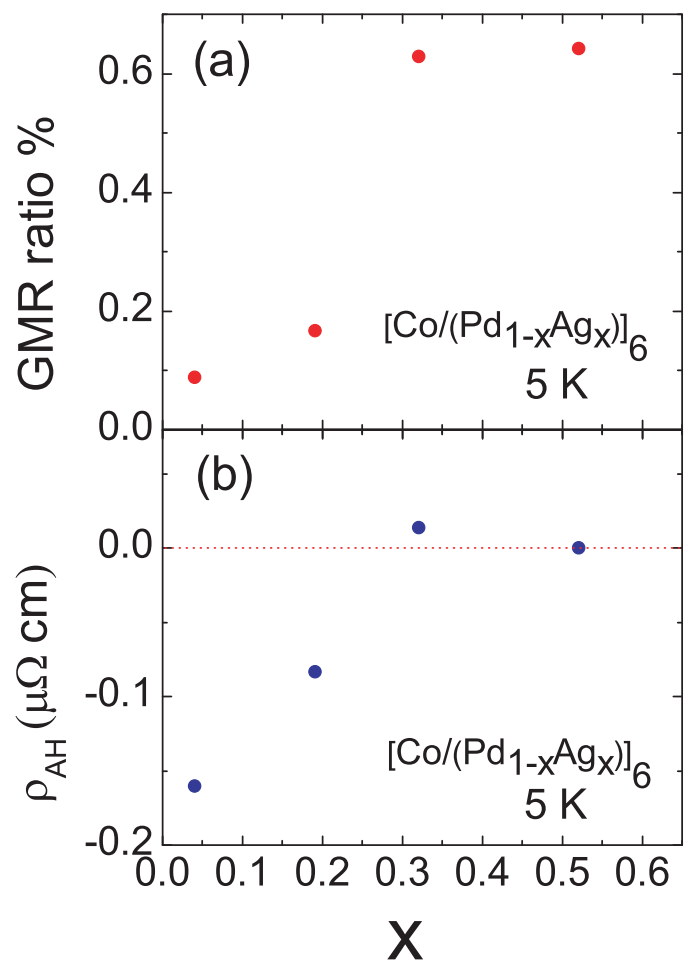

FIG. 4. (a) GMR ratio and (b) $\rho_{A H}$ as a function of $x$ at $5 \mathrm{~K}$ for $[\operatorname{Co}(3 \AA) /$ $\left.\operatorname{Pd}_{1-x} \mathrm{Ag}_{x}(5 \AA)\right]_{6}$, respectively. transition from surface scattering-dominated to interface scattering-dominated AHE. The theoretical models, which have successfully explained the GMR effect, mainly consider spin-dependent interface scattering in the direction normal to the film plane. ${ }^{21,22}$ How the spin-dependent scattering at the interface in the transverse direction, which induces AHE, is still missing.

The present study indicates, with the increase of the substitution of Pd with Ag at $x \geq 0.32$, the decrease in $\rho_{x x}$ results in an increase in mean free path, which is expected to enhance the contribution of spin-dependent surface scattering to the spin transport, but the contribution of spindependent interface scattering is enhanced as well, the latter induces co-occurrence of the enhancement in GMR [Fig. 4(a)] and interface scattering-dominated AHE overwhelming AHE from surface scattering [inset in Fig. 2(a)].

Finally, we carried out scaling analysis of the interface scattering contribution on AHE in the multilayers. In order to eliminate surface scattering contribution on AHE, we fabricated the multilayers with repetition up to 80 . The temperature dependence of $\rho_{A H}$ for $\left[\mathrm{Co} / \mathrm{Pd}_{1-x} \mathrm{Ag}_{x}\right]_{80}$ is shown in the inset in Fig. 5. Compared with those of multilayers with $n=6$, as shown in Fig. 2(a), $\rho_{A H}$ in the multilayers with $n=80$ is obviously larger than those with $n=6$, in particular, $\rho_{A H}$ changes from negative to positive for $x=0$, and for $\mathrm{x}=0.04$ and 0.19 in temperatures above 150 and $100 \mathrm{~K}$, respectively, which should be due to the reduction in surface scattering contribution to AHE.

It has been observed that the surface scattering contribution to AHE could be ignored in $x=0$, i.e., in $[\mathrm{Co} / \mathrm{Pd}]_{80}$ multilayer. ${ }^{13}$ However, with increasing temperature, the sign change in $\rho_{A H}$ from negative to positive in $x=0.04$ and 0.19 , and downward curvature in $x=0.32$ (inset in Fig. 5), indicate that the surface scattering contribution to AHE is still present in these samples. Scaling analysis would fail without subtraction of the surface scattering contribution from the total AHE. ${ }^{13}$ However, for $x=0.52, \rho_{A H}$ is positive, and it increases with increasing temperature (inset in Fig. 5). On the other hand, the relatively large MR ratio of this film

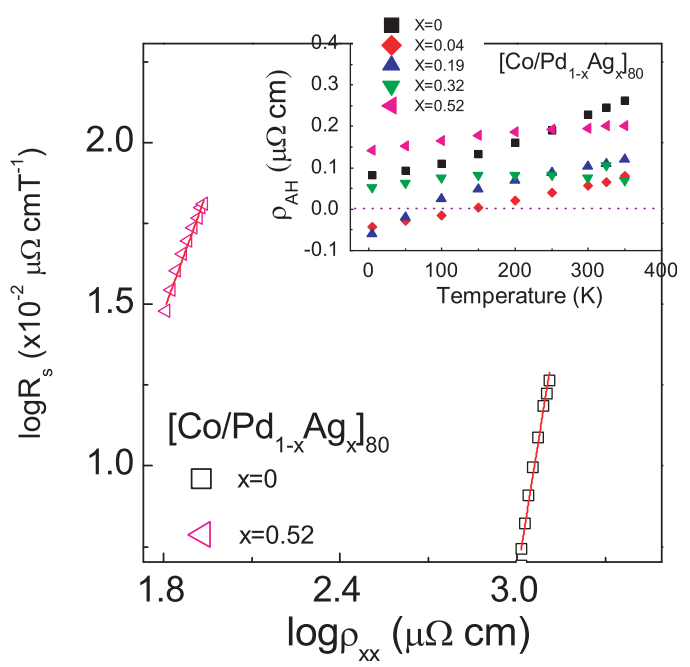

FIG. 5. The plot of $\log R_{s}$ vs $\log \rho_{x x}$ for the $\left[\mathrm{Co}(3 \AA) / \mathrm{Pd}_{1-x} \mathrm{Ag}_{x}(5 \AA)\right]_{80}$ multilayers with $x=0$ and 0.52 . The lines are linear fittings to the data. Inset: The temperature dependence of $\rho_{A H}$ for the $\left[\mathrm{Co}(3 \AA) / \mathrm{Pd}_{1-x} \mathrm{Ag}_{x}(5 \AA)\right]_{80}$ multilayers with $x=0,0.04,0.19,0.32$, and 0.52 . 
indicates that a strong spin-dependent interface scattering is presented. Therefore, we tried to process the scaling analysis for this sample by Eq. (2). The result of $\log R_{s}$ vs $\log \rho_{x x}$ is shown in the main panel in Fig. 5. For comparison, that of $x=0$ is also presented. A linear fit for the data of $x=0.52$ leads to $\gamma=3.44 \pm 0.13$, and the film with $x=0$ gives $\gamma=5.71 \pm 0.25$. The difference in the magnetotransport properties in these two multilayers is worth noticing. The sample of $x=0$ exhibits an AMR behavior arising from spinorbit coupling, while the sample with $x=0.52$ exhibits a GMR behavior. How these distinguished transport properties affect AHE, thus the different $\gamma$ values, is still not clear.

In summary, we studied $\mathrm{AHE}$ and magnetoresistance behavior in $\left[\mathrm{Co} / \mathrm{Pd}_{1-x} \mathrm{Ag}_{x}\right]_{n}$ multilayers. All these samples present perpendicular magnetic anisotropy. For the multilayers with $n=6$, the AHE sign change from negative to positive has been attributed to the $\mathrm{Ag}$ substitution induced enhancement in the interface scattering contribution to AHE, while weakening the surface scattering contribution, though longitudinal resistivity was found to decrease in high Ag content. The enhancement in interface scattering contribution to AHE was associated with the increase of giant magnetoresistance. For the multilayers with $n=80$, scaling analysis yields $\gamma \sim 3.44$ for the sample with $x=0.52$, which exhibits a GMRtype transport behavior. In contrast, $\gamma \sim 5.7$ for the sample with $x=0$ which presents an AMR-type transport behavior.

We wish to thank Q. Zhang, R. O. Aboljadayel, Y. B. Yao, and L. Chen for sample fabrications and valuable discussions.
${ }^{1}$ E. H. Hall, Am. J. Math. 2, 287 (1879).

${ }^{2}$ N. Nagaosa, J. Sinova, S. Onoda, A. H. MacDonald, and N. P. Ong, Rev. Mod. Phys. 82, 1539 (2010).

${ }^{3}$ L. Berger and G. Bergmann, The Hall Effect and Its Applications, edited by C. L. Chien and C. R. Westgate (Plenum, New York, 1980).

${ }^{4} \mathrm{C}$. M. Hurd, The Hall Effect in Metals and Alloys (Plenum, New York, 1972).

${ }^{5}$ J. Smit, Physica (Amsterdam) 21, 877 (1955).

${ }^{6}$ L. Berger, Phys. Rev. B 2, 4559 (1970).

${ }^{7}$ R. Karplus and J. M. Luttinger, Phys. Rev. 95, 1154 (1954).

${ }^{8}$ Z. Fang, N. Nagaosa, K. S. Tahakashi, A. Asamitsu, R. Mathieu, T. Ogasawara, H. Yamada, M. Kawasaki, Y. Tokura, and K. Terakura, Science 302, 92 (2003).

${ }^{9}$ Y. Shiomi, Y. Onose, and Y. Tokura, Phys. Rev. B 79, 100404(R) (2009).

${ }^{10}$ Y. Tian, L. Ye, and X. Jin, Phys. Rev. Lett. 103, 087206 (2009).

${ }^{11}$ S. N. Song, C. Sellers, and J. B. Ketterson, Appl. Phys. Lett. 59, 479 (1991).

${ }^{12}$ C. L. Canedy, X. W. Li, and G. Xiao, Phys. Rev. B 62, 508 (2000).

${ }^{13}$ Z. B. Guo, W. B. Mi, R. O. Aboljadayel, B. Zhang, Q. Zhang, P. G. Barba, A. Manchon, and X. X. Zhang, Phys. Rev. B 86, 104433 (2012).

${ }^{14}$ P. Xiong, G. Xiao, J. Q. Wang, J. Q. Xiao, J. S. Jiang, and C. L. Chien, Phys. Rev. Lett. 69, 3220 (1992).

${ }^{15}$ A. Gerber, A. Milner, A. Finkler, M. Karpovski, L. Goldsmith, J. TuaillonCombes, O. Boisron, P. Mélinon, and A. Perez, Phys. Rev. B 69, 224403 (2004).

${ }^{16}$ Z. B. Guo, W. B. Mi, Q. Zhang, B. Zhang, R. O. Aboljadayel, and X. X. Zhang, Solid State Commun. 152, 220 (2012).

${ }^{17}$ D. Rosenblatt. M. Karpovski, and A. Gerber, Appl. Phys. Lett. 96, 022512 (2010).

${ }^{18}$ S. S. Bogomolov, R. J. Highmore, W. C. Shih, A. L. Greer, R. E. Somekh, and J. E. Evetts, J. Appl. Phys. 73, 6841 (1993).

${ }^{19}$ B. N. Engel, C. D. England, R. A. Van Leeuwen, M. H. Wiedmann, and C. M. Falco, Phys. Rev. Lett. 67, 1910 (1991).

${ }^{20}$ L. Néel, J. Phys. Radium 15, 376 (1954).

${ }^{21}$ R. E. Cemley and J. Barnaś, Phys. Rev. Lett. 63, 664 (1989).

${ }^{22}$ A. Barthélémy and A. Fert, Phys. Rev. B 43, 13124 (1991). 